\title{
Quandle coloring quivers of links using dihedral quandles*
}

\author{
Yuta Taniguchi
}

\section{Introduction}

A quandle $([7,8])$ is an algebraic structure defined on a set with a binary operation whose definition was motivated from knot theory. D. Joyce and S. V. Matveev [7,8 associated a quandle to a link, which is so-called the link quandle or the fundamental quandle of a link. Since then many link invariants using link quandles have been introduced and studied. A typical and elementary example is the quandle coloring number which is the cardinal number of the set of quandle homomorphisms from the link quandle to a fixed finite quandle. A quandle cocycle invariant [1] and a shadow quandle cocycle invariant (cf. [4]) are also such link invariants, which are enhancements of the quandle coloring number.

In 2019, K. Cho and S. Nelson [5] introduced the notion of a quandle coloring quiver, which is a quiver-valued link invariant, and gave interesting examples. This invariant is defined when we fix a finite quandle and a set of its endomorphisms. They also introduced in [6] the notion of a quandle cocycle quiver which is an enhancement of the quandle coloring quiver by assigning to each vertex a weight computed using a quandle 2-cocycle.

In this paper, we study quandle coloring quivers using dihedral quandles. We show that, when we use a dihedral quandle of prime order, the quandle coloring quivers are equivalent to the quandle coloring numbers (Theorem 3.3. . The notions of a quandle coloring quiver and a quandle cocyle quiver

\footnotetext{
*keywords: quandle, quandle coloring quiver, dihedral quandle Mathematics Subject Classification 2010: 57M25, 57M27

Mathematics Subject Classification 2020: 57K10, 57K12
} 
are naturally generalized to a shadow quandle coloring quiver and a shadow quandle cocycle quiver. We show that, when we use a dihedral quandle of prime order and Mochizuki's 3-cocycle, the shadow quandle cocycle quivers are equivalent to the shadow quandle cocycle invariants (Theorem 6.1).

This paper is organaized as follows. In Section 2, we recall the definition of a quandle, a quandle coloring and a shadow quandle cocycle invariant. In Section 3, we recall the definition of the quandle coloring quiver of a link, and discuss quandle coloring quivers using a dihedral quandle of prime order. In Section 4, we discuss quandle coloring quivers of a dihedral quandle of composite order. In Section 5, we introduce the notion of a shadow quandle coloring quiver and a shadow quandle cocycle quiver. In Section 6, we discuss shadow quandle cocycle quivers using a dihedral quandle of prime order and Mochizuki's 3-cocycle.

\section{Quandles and quandle cocycle invariants}

\subsection{Quandles}

A quandle is a set $X$ with a binary operation $*: X \times X \rightarrow X$ satisfying the following three axioms.

(Q1) For any $x \in X$, we have $x * x=x$.

(Q2) For any $y \in X$, the map $* y: X \rightarrow X, x \mapsto x * y$ is a bijection.

(Q3) For any $x, y, z \in X$, we have $(x * y) * z=(x * z) *(y * z)$.

These axioms correspond to the three kinds of Reidemeister moves (cf. [7,8]).

Example 2.1. The dihedral quandle of order $n$, denoted by $R_{n}$, is $\mathbb{Z}_{n}=\mathbb{Z} / n \mathbb{Z}$ with an operation $*$ defined by $x * y=2 y-x$.

Example 2.2. Let $M$ be a $\mathbb{Z}\left[t^{ \pm 1}\right]$-module. We define an operation by $x * y=$ $t x+(1-t) y$. Then, $M$ is a quandle, which is called an Alexander quandle.

A map $f: X \rightarrow Y$ between quandles is called a (quandle) homomorphism if $f(x * y)=f(x) * f(y)$ for any $x, y \in X$. A quandle homomorphism $f: X \rightarrow Y$ is a quandle isomorphism, a quandle endomorphism, or a quandle automorphism if it is a bijection, if $X=Y$, or if $X=Y$ and it is a bijection, respectively. We denote by $\operatorname{Hom}(X, Y)$ the set of quandle homomorphisms from $X$ to $Y$, by $\operatorname{End}(X)$ the set of quandle endomorphisms of $X$ and by $\operatorname{Aut}(X)$ the set of quandle automorphisms of $X$. 


\subsection{Quandle colorings and shadow cocycle invariants}

Let $X$ be a quandle and $D$ be an oriented link diagram on $\mathbb{R}^{2}$. We denote the set of the $\operatorname{arcs}$ of $D$ by $\operatorname{Arc}(D)$. A map $c: \operatorname{Arc}(D) \rightarrow X$ is an $X$-coloring if $c$ satisfies following condition at every crossing of $D$.

- Let $x_{i}, x_{j}, x_{k}$ be arcs around a crossing as in Figure 1 (left). Then, $c\left(x_{i}\right) * c\left(x_{j}\right)=c\left(x_{k}\right)$.

The value $c(a)$ assigned to arc $a$ is called the label. An $X$-coloring $c$ is a trivial coloring if $\mathrm{c}$ is a constant map. We denote by $\operatorname{Col}_{X}(D)$ the set of $X$-colorings of $D$.

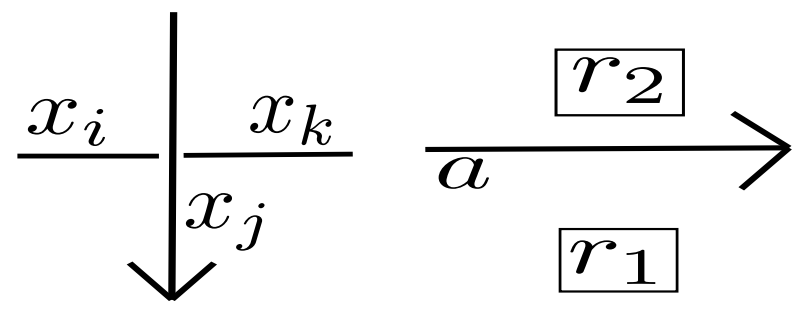

Figure 1: coloring condition.

If two link diagrams $D$ and $D^{\prime}$ are related by Reidemeister moves, we have a bijection between $\operatorname{Col}_{X}(D)$ and $\operatorname{Col}_{X}\left(D^{\prime}\right)$. Thus, when $X$ is a finite quandle, the cardinal number $\left|\mathrm{Col}_{X}(D)\right|$ of $\mathrm{Col}_{X}(D)$ is a link invariant. It is called the $X$-coloring number or the coloring number by $X$.

Let $|D|$ be immersed circles in $\mathbb{R}^{2}$ obtained from $D$ by ignoring over/under information of the crossings. We denote by Region $(D)$ the set of connected component of $\mathbb{R}^{2} \backslash|D|$ and by $r_{\infty}$ the unbounded region of $\operatorname{Region}(D)$. A map $c_{*}: \operatorname{Arc}(D) \cup \operatorname{Region}(D) \rightarrow X$ is a shadow $X$-coloring if $c_{*}$ satisfies the following conditions.

- $\left.c_{*}\right|_{\operatorname{Arc}(D)}$ is an $X$-coloring.

- Let $r_{1}$ and $r_{2}$ be adjacent regions of $D$ along an arc $a$ as in Figure 1 (right). Then, $c_{*}\left(r_{1}\right) * c_{*}(a)=c_{*}\left(r_{2}\right)$.

We denote by $\mathrm{SCol}_{X}(D)$ the set of shadow $X$-colorings of $D$ and by $\operatorname{SCol}_{X}(D, a)$ the set of shadow $X$-colorings of $D$ which satisfy $c_{*}\left(r_{\infty}\right)=a$ for $a \in X$.

Let $A$ be an abelian group. A map $\theta: X^{3} \rightarrow A$ is called a quandle 3 -cocycle if $\theta$ satisfies the following conditions (cf. [1]).

- For any $x, y \in X, \theta(x, x, y)=\theta(x, y, y)=0$. 
- For any $x, y, z, w \in X, \theta(x, y, z)+\theta(x * z, y * z, w)+\theta(x, z, w)=\theta(x *$ $y, z, w)+\theta(x, y, w)+\theta(x * w, y * w, z * w)$.

Example 2.3. ([9, 10]) Let $p$ be an odd prime. We define a map $\theta_{p}:\left(R_{p}\right)^{3} \rightarrow$ $\mathbb{Z}_{p}$ by

$$
\theta_{p}(x, y, z)=(x-y) \frac{\left(y^{p}+(2 y-z)^{p}-2 z^{p}\right)}{p} .
$$

Then, $\theta_{p}$ is a quandle 3-cocycle, which is referred to as Mochizuki's 3-cocycle in this paper.

Let $\theta: X^{3} \rightarrow A$ be a quandle 3-cocycle of a quandle $X$ and $D$ be an oriented link diagram. For a shadow $X$-coloring $c_{*}$ of $D$, we associated a weight $\pm \theta(x, y, z)$ to each crossing of $D$, where $x \in X$ is the label of the region and $y, z \in X$ are labels of the arcs indicated in Figure 2. Then, we sum up the weights all over the crossings of $D$ to obtain an element of $A$ denoted by $\Phi_{\theta}\left(D, c_{*}\right)$.
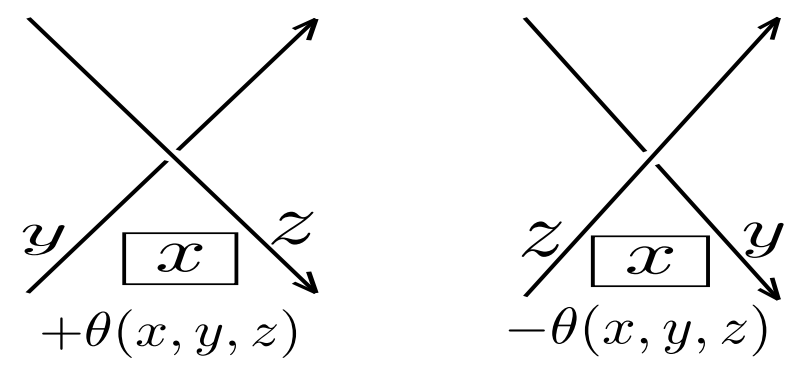

Figure 2: The weight of a crossing.

When $X$ is a finite quandle, $\Phi_{\theta}(D)=\left\{\Phi_{\theta}\left(D, c_{*}\right) \mid c_{*} \in \operatorname{SCol}_{X}(D)\right\}$ as a multiset is a link invariant, which we call a shadow quandle cocycle invariant (cf. 3]).

\section{Quandle coloring quivers using a dihedral quandle of prime order}

Let $X$ be a finite quandle and $D$ be an oriented link diagram. For any subset $S \subset \operatorname{End}(X)$, the quandle coloring quiver of $D$, which is denoted by $Q_{X}^{S}(D)$, 
is the quiver with a vertex for each $X$-coloring $c \in \mathrm{Col}_{X}(D)$ and an edge directed from $v$ to $w$ when $w=f \circ v$ for an element $f \in S$. In [5], it is proved that $Q_{X}^{S}(D)$ is a link invariant. Precisely speaking, if two link diagrams $D$ and $D^{\prime}$ are related by Reidemeister moves, then the quandle coloring quivers $Q_{X}^{S}(D)$ and $Q_{X}^{S}\left(D^{\prime}\right)$ are isomorphic as quivers for any $S \subset \operatorname{End}(X)$.

Remark 3.1. Note that the quandle coloring number of $D$ by using a finite quandle $X$ is $\left|\operatorname{Col}_{X}(D)\right|$, which is the number of vertices of $Q_{X}^{S}(D)$. Thus, the quandle coloring quiver $Q_{X}^{S}(D)$ is in general a stronger link invariant than the quandle coloring number. For example, both of the knots $8_{10}$ and $8_{18}$ have the same quandle coloring number by using the dihedral quandle $R_{9}$ of order 9 , which is 81 . On the other hand, their quandle coloring quivers using $R_{9}$ with $S=\operatorname{End}\left(R_{9}\right)$ are not isomorphic as quivers (Example 11 of [5]).

Lemma 3.2. Let $X$ be a finite quandle and let $D$ and $D^{\prime}$ be oriented link diagrams. Assume that there exists a bijection $\varphi: \operatorname{Col}_{X}(D) \rightarrow \operatorname{Col}_{X}\left(D^{\prime}\right)$ which satisfies the following condition, referred to as the condition $(\alpha)$,

- For any $f \in \operatorname{End}(X)$ and $c \in \mathrm{Col}_{X}(D), f \circ \varphi(c)=\varphi(f \circ c)$.

Then the quandle coloring quivers $Q_{X}^{S}(D)$ and $Q_{X}^{S}\left(D^{\prime}\right)$ are isomorphic for any $S \subset \operatorname{End}(X)$.

Proof. If $(v, w)$ is an edge of $Q_{X}^{S}(D)$, we have

$$
\begin{aligned}
\varphi(w) & =\varphi(f \circ v) \\
& =f \circ \varphi(v)
\end{aligned}
$$

for some $f \in S$. Then, $(\varphi(v), \varphi(w))$ is an edge of $Q_{X}^{S}\left(D^{\prime}\right)$. As the same way, it holds that $(v, w)$ is an edge of $Q_{X}^{S}(D)$ if and only if $(\varphi(v), \varphi(w))$ is an edge of $Q_{X}^{S}\left(D^{\prime}\right)$. Therefore, $\varphi$ is an isomorphism between $Q_{X}^{S}(D)$ and $Q_{X}^{S}\left(D^{\prime}\right)$.

The following result is one of the main results of this paper.

Theorem 3.3. Let $D$ and $D^{\prime}$ be oriented link diagrams and $p$ be a prime. For any $S \subset \operatorname{End}\left(R_{p}\right)$, the quandle coloring quivers $Q_{R_{p}}^{S}(D)$ and $Q_{R_{p}}^{S}\left(D^{\prime}\right)$ are isomorphic if and only if $\left|\mathrm{Col}_{R_{p}}(D)\right|=\left|\mathrm{Col}_{R_{p}}\left(D^{\prime}\right)\right|$.

Lemma 3.4. Let $n$ be a positive integer greater than 1 . If $f: R_{n} \rightarrow R_{n}$ is a quandle homomorphism, then there exist unique elements $a, b \in \mathbb{Z}_{n}$ such that $f(x)=a x+b$ for any $x \in X$. 
Proof. Put $a=f(1)-f(0)$ and $b=f(0)$. We define a map $g: R_{n} \rightarrow R_{n}$ by $g(x)=a x+b$. It is seen by a directed calculation that $g$ is a quandle homomorphism. By definition of $g$, we have $f(0)=g(0)$ and $f(1)=g(1)$. Recall that $(m-1) * m=m+1$ for any $m \in R_{n}$, and we have $g(m)=f(m)$ for any $m \in R_{n}$. The uniqueness of $a$ and $b$ is seen by evaluating $f$ with 0 and 1.

Remark 3.5. If $f$ is a quandle automorphism of $R_{n}$, then $a$ is an element of $\mathbb{Z}_{n}^{\times}$. When $p$ is a prime, we have $\operatorname{End}\left(R_{p}\right)=\operatorname{Aut}\left(R_{p}\right) \cup\{$ constant maps $\}$.

Proof of Theorem 3.3. The only if part is trivial. We consider the if part.

We show that there exists a bijection $\varphi: \operatorname{Col}_{R_{p}}(D) \rightarrow \operatorname{Col}_{R_{p}}\left(D^{\prime}\right)$ which satisfies the condition $(\alpha)$ in Lemma 3.2 .

Recall that $\mathrm{Col}_{R_{p}}(D)$ and $\mathrm{Col}_{R_{p}}\left(D^{\prime}\right)$ are $\mathbb{Z}_{p}$-vector spaces. By assumption, they have the same cardinality and hence their dimensions are the same, say $n$. Take a basis $\left\{c_{1}, \ldots, c_{n}\right\}$ of $\operatorname{Col}_{R_{p}}(D)$ and a basis $\left\{c_{1}^{\prime}, \ldots, c_{n}^{\prime}\right\}$ of $\mathrm{Col}_{R_{p}}\left(D^{\prime}\right)$ such that $c_{1}$ and $c_{1}^{\prime}$ are the trivial coloring which is the constant map onto 1. Let $f$ be a quandle endomorphism of $R_{p}$. By Lemma 3.4, we have $f(x)=a x+b$ for some $a, b \in \mathbb{Z}_{p}$. Then,

$$
\begin{aligned}
f \circ c(x) & =f(c(x)) \\
& =a c(x)+b \\
& =a c(x)+b c_{1}(x)
\end{aligned}
$$

for any $c \in \operatorname{Col}_{R_{p}}(D)$ and $x \in \operatorname{Arc}(D)$. Therefore, we have $f \circ c=a c+b c_{1}$ for any $c \in \operatorname{Col}_{R_{p}}(D)$. Similarly, we can show that $f \circ c^{\prime}=a c^{\prime}+b c_{1}^{\prime}$ for any $c^{\prime} \in \mathrm{Col}_{R_{p}}\left(D^{\prime}\right)$.

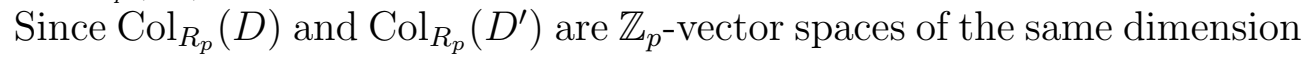
$n$, there is a $\mathbb{Z}_{p}$-linear isomorphism $\varphi: \operatorname{Col}_{R_{p}}(D) \rightarrow \mathrm{Col}_{R_{p}}\left(D^{\prime}\right)$ such that $\varphi\left(c_{1}\right)=c_{1}^{\prime}$. Then,

$$
\begin{aligned}
\varphi(f \circ c) & =\varphi\left(a c+b c_{1}\right) \\
& =a \varphi(c)+b \varphi\left(c_{1}\right) \\
& =a \varphi(c)+b c_{1}^{\prime} \\
& =f \circ \varphi(c) .
\end{aligned}
$$

Therefore, $\varphi$ satisfies the condition $(\alpha)$. 


\section{Quandle coloring quivers using a dihedral quandle of composite order}

We discuss quandle coloring quivers using a dihedral quandle of composite order.

Theorem 4.1. Let $D$ and $D^{\prime}$ be oriented link diagrams and let $m$ and $n$ be coprime integers greater than 1. Suppose that there exists bijections $\varphi_{m}$ : $\mathrm{Col}_{R_{m}}(D) \rightarrow \mathrm{Col}_{R_{m}}\left(D^{\prime}\right)$ and $\varphi_{n}: \mathrm{Col}_{R_{n}}(D) \rightarrow \mathrm{Col}_{R_{n}}\left(D^{\prime}\right)$ which satisfy the condition $(\alpha)$. Then $Q_{R_{m n}}^{S}(D)$ and $Q_{R_{m n}}^{S}\left(D^{\prime}\right)$ are isomorphic for any $S \subset$ $\operatorname{End}\left(R_{m n}\right)$.

Proof. Since $m$ and $n$ are coprime, by Chinese remainder theorem, we have a bijection $R_{m n} \rightarrow R_{m} \times R_{n}$ by $[x]_{m n} \mapsto\left([x]_{m},[x]_{n}\right)$, where $[x]_{m}$ means an element of $R_{m}=\mathbb{Z} / m \mathbb{Z}$ represented by an integer $x$. It is not only a group isomorphism but also a quandle isomorphism. Thus $R_{m n}$ can be identified with $R_{m} \times R_{n}$ as a quandle. On the other hand, we can naturally idenitfy $\mathrm{Col}_{R_{m} \times R_{n}}(D)$ with $\mathrm{Col}_{R_{m}}(D) \times \mathrm{Col}_{R_{n}}(D)$. Then, we have a natural bijection

$$
\psi: \mathrm{Col}_{R_{m n}}(D) \rightarrow \mathrm{Col}_{R_{m}}(D) \times \mathrm{Col}_{R_{n}}(D) .
$$

And similarly we have a natural bijection $\psi^{\prime}: \operatorname{Col}_{R_{m n}}\left(D^{\prime}\right) \rightarrow \operatorname{Col}_{R_{m}}\left(D^{\prime}\right) \times$ $\mathrm{Col}_{R_{n}}\left(D^{\prime}\right)$.

Let us define a map

$$
\psi_{m}: \operatorname{End}\left(R_{m n}\right) \rightarrow \operatorname{End}\left(R_{m}\right)
$$

by $\psi_{m}(f)\left([x]_{m}\right)=[a]_{m}[x]_{m}+[b]_{m}$ for any $f \in \operatorname{End}\left(R_{m n}\right)$, where $[a]_{m},[b]_{m} \in$ $R_{m}$ are images of $[a]_{m n},[b]_{m n} \in R_{m n}$ which are uniquely determined from $f$ by Lemma 3.4 with $f\left([x]_{m n}\right)=[a]_{m n}[x]_{m n}+[b]_{m n}$ for $[x]_{m n} \in R_{m n}$. Similarly, we define a map $\psi_{n}: \operatorname{End}\left(R_{m n}\right) \rightarrow \operatorname{End}\left(R_{n}\right)$. By a direct calculation, we see that $\psi(f \circ c)=\left(\psi_{m}(f) \times \psi_{n}(f)\right) \circ(\psi(c))$ and $\psi^{\prime}\left(f \circ c^{\prime}\right)=\left(\psi_{m}(f) \times \psi_{n}(f)\right) \circ\left(\psi\left(c^{\prime}\right)\right)$ for any $f \in \operatorname{End}\left(R_{m n}\right), c \in \mathrm{Col}_{R_{m n}}(D)$ and $c^{\prime} \in \mathrm{Col}_{R_{m n}}\left(D^{\prime}\right)$.

By assumption, there exists bijections $\varphi_{m}: \mathrm{Col}_{R_{m}}(D) \rightarrow \mathrm{Col}_{R_{m}}\left(D^{\prime}\right)$ and $\varphi_{n}: \mathrm{Col}_{R_{n}}(D) \rightarrow \operatorname{Col}_{R_{n}}\left(D^{\prime}\right)$ which satisfy the condition $(\alpha)$. Let $\varphi=\varphi_{m} \times$ $\varphi_{n}: \mathrm{Col}_{R_{m}}(D) \times \mathrm{Col}_{R_{n}}(D) \rightarrow \mathrm{Col}_{R_{m}}\left(D^{\prime}\right) \times \mathrm{Col}_{R_{n}}\left(D^{\prime}\right)$ and define a bijection $\Psi$ by $\Psi=\left(\psi^{\prime}\right)^{-1} \circ \varphi \circ \psi: \operatorname{Col}_{R_{m n}}(D) \rightarrow \operatorname{Col}_{R_{m n}}\left(D^{\prime}\right)$. Then, we have

$$
\begin{aligned}
\Psi(f \circ c) & =\left(\psi^{\prime}\right)^{-1} \circ \varphi\left(\left(\psi_{m}(f) \times \psi_{n}(f)\right) \circ(\psi(c))\right) \\
& =\left(\psi^{\prime}\right)^{-1}\left(\left(\psi_{m}(f) \times \psi_{n}(f)\right) \circ(\varphi \circ \psi(c))\right) \\
& =f \circ \Psi(c)
\end{aligned}
$$


for any $f \in \operatorname{End}\left(R_{m n}\right)$ and $c \in \operatorname{Col}_{R_{m n}}(D)$. Therefore, $\Psi$ satisfies the condition $(\alpha)$, which implies by Lemma 3.2 the assertion.

Corollary 4.2. Let $D$ and $D^{\prime}$ be oriented link diagrams and $P=p_{1}^{e_{1}} p_{2}^{e_{2}} \ldots p_{n}^{e_{n}}$ be the prime factorization of a positive integer $P$. If there exists a bijection $\varphi_{p_{i}^{e_{i}}}: \mathrm{Col}_{R_{p_{i}}}(D) \rightarrow \mathrm{Col}_{R_{p_{i}}}\left(D^{\prime}\right)$ which satisfies the condition $(\alpha)$ for each $i$, then $Q_{R_{P}}^{S}(D)$ and $Q_{R_{P}}^{S}\left(D^{\prime}\right)$ are isomorphic for any $S \subset \operatorname{End}\left(R_{P}\right)$.

Proof. By assumption, there exists bijections $\varphi_{p_{1}^{e_{1}}}: \mathrm{Col}_{R_{p_{1} e_{1}}}(D) \rightarrow \operatorname{Col}_{R_{p_{1}}}\left(D^{\prime}\right)$ and $\varphi_{p_{2} e_{2}}: \operatorname{Col}_{R_{p_{2}}}(D) \rightarrow \operatorname{Col}_{R_{p_{2}}}\left(D^{\prime}\right)$. As seen in the proof of Theorem 4.1. we have a bijection $\varphi_{p_{1}^{e_{1}} p_{2}^{e_{2}}}: \operatorname{Col}_{R_{p_{1} p_{2} e_{2}}}(D) \rightarrow \mathrm{Col}_{R_{p_{1} p_{1} e_{2}}}\left(D^{\prime}\right)$ which satisfies the condition $(\alpha)$. Repeating this procedure, we have a bijection $\varphi_{P}: \mathrm{Col}_{R_{P}}(D) \rightarrow \mathrm{Col}_{R_{P}}\left(D^{\prime}\right)$ which satisfies the condition $(\alpha)$, which implies by Lemma 3.2 the assertion.

Theorem 4.3. Let $D$ and $D^{\prime}$ be oriented link diagrams and $P=p_{1} p_{2} \ldots p_{n}$ be a positive integer for some distinct primes $p_{1}, \ldots, p_{n}$. For any $S \subset \operatorname{End}\left(R_{P}\right)$, the quandle coloring quivers $Q_{R_{P}}^{S}(D)$ and $Q_{R_{P}}^{S}\left(D^{\prime}\right)$ are isomorphic if and only if $\left|\mathrm{Col}_{R_{P}}(D)\right|=\left|\mathrm{Col}_{R_{P}}\left(D^{\prime}\right)\right|$.

Proof. The only if part is trivial. We show the if part. Suppose that $\left|\mathrm{Col}_{R_{P}}(D)\right|=\left|\mathrm{Col}_{R_{P}}\left(D^{\prime}\right)\right|$. As seen in the proof of Theorem 4.1. $\mathrm{Col}_{R_{P}}(D)$ is naturally identified with $\prod_{i=1}^{n} \operatorname{Col}_{R_{p_{i}}}(D)$. Since $\left|\operatorname{Col}_{R_{P}}(D)\right|=\left|\operatorname{Col}_{R_{P}}\left(D^{\prime}\right)\right|$, we have $\left|\mathrm{Col}_{R_{p_{i}}}(D)\right|=\left|\mathrm{Col}_{R_{p_{i}}}\left(D^{\prime}\right)\right|$ for each $i$. As seen in the proof of Theorem 3.3, we have a bijection $\varphi_{p_{i}}: \mathrm{Col}_{R_{p_{i}}}(D) \rightarrow \mathrm{Col}_{R_{p_{i}}}\left(D^{\prime}\right)$ which satisfies the condition $(\alpha)$ for each $i$. By Corollary 4.2 , we have a bijection $\varphi_{P}: \operatorname{Col}_{R_{P}}(D) \rightarrow \operatorname{Col}_{R_{P}}\left(D^{\prime}\right)$ which satisfies the condition $(\alpha)$, which implies by Lemma 3.2 the assertion.

\section{Shadow versions of quandle quivers}

In this section we define the shadow quandle coloring quiver and the shadow quandle cocycle quiver, which are shadow versions of the quandle coloring quiver and the quandle cocycle quiver defined by Cho and Nelson [5, 6].

Definition 5.1. Fix a finite quandle $X$, an element $a \in X$ and a subset $S \subset \operatorname{End}(X)$. The shadow quandle coloring quiver of an oriented link diagram $D$, which is denoted by $S Q_{X}^{S}(D, a)$, is the quiver with a vertex for each 
shadow $X$-coloring $c_{*} \in \operatorname{SCol}_{X}(D, a)$ and an edge directed from $v_{*}$ to $w_{*}$ when $\left.w_{*}\right|_{\operatorname{Arc}(D)}=\left.f \circ v_{*}\right|_{\operatorname{Arc}(D)}$ for an element $f \in S$.

The shadow quandle coloring quiver is a link invariant. However, it is nothing more than the quandle coloring quiver as seen below.

Proposition 5.2. The quandle coloring quiver $Q_{X}^{S}(D)$ and the shadow quandle coloring quiver $S Q_{X}^{S}(D, a)$ are isomorphic for any $S \subset \operatorname{End}(X)$ and $a \in X$.

Proof. For any $a \in X$ and $X$-coloring $c \in \operatorname{Col}_{X}(D)$, there is a unique shadow $X$-coloring $c_{*}$ such that $\left.c_{*}\right|_{\operatorname{Arc}(D)}=c$ and $c_{*}\left(r_{\infty}\right)=a$ (cf. [3|). Hence, we have a natural bijection $\varphi: \operatorname{Col}_{X}(D) \rightarrow \operatorname{SCol}_{X}(D, a)$. By definition of the shadow quandle coloring quiver, we see that $\varphi$ is a quiver isomorphism between $Q_{X}^{S}(D)$ and $S Q_{X}^{S}(D, a)$ for any subset $S \subset \operatorname{End}(X)$.

Next, we define the shadow quandle cocycle quiver.

Definition 5.3. Fix a finite quandle $X$, an element $a \in X$, a subset $S \subset$ $\operatorname{End}(X)$, an abelian group $A$ and a quandle 3-cocycle $\theta: X^{3} \rightarrow A$. The shadow quandle cocycle quiver of an oriented link diagram $D$, which is denoted by $S Q_{X}^{S, \theta}(D, a)$, is the pair $\left(S Q_{X}^{S}(D, a), \rho\right)$ of the shadow quandle coloring quiver $S Q_{X}^{S}(D, a)$ and a map $\rho: \operatorname{SCol}_{X}(D, a) \rightarrow A$ defined by $\rho\left(c_{*}\right)=\Phi_{\theta}\left(D, c_{*}\right)$.

Definition 5.4. Let $S Q_{X}^{S, \theta}(D, a)=\left(S Q_{X}^{S}(D, a), \rho\right)$ be the shadow quandle cocycle quiver of an oriented link diagram $D$ and let $S Q_{X}^{S, \theta}\left(D^{\prime}, a\right)=$ $\left(S Q_{X}^{S}\left(D^{\prime}, a\right), \rho^{\prime}\right)$ be that of $D^{\prime}$. We say that $S Q_{X}^{S, \theta}(D, a)$ and $S Q_{X}^{S, \theta}\left(D^{\prime}, a\right)$ are isomorphic if there is a bijection $\varphi: \mathrm{SCol}_{X}(D, a) \rightarrow \mathrm{SCol}_{X}\left(D^{\prime}, a\right)$ which satisfies the following conditions.

- $\varphi$ determines a quiver isomorphism from $S Q_{X}^{S}(D, a)$ to $S Q_{X}^{S}\left(D^{\prime}, a\right)$.

- For any $c_{*} \in \operatorname{SCol}_{X}(D, a)$, we have $\rho\left(c_{*}\right)=\rho^{\prime}\left(\varphi\left(c_{*}\right)\right)$.

Proposition 5.5. The shadow quandle cocycle quiver is a link invariant. Namely, for oriented link diagrams $D$ and $D^{\prime}$ which are related by Reidemeister moves, the shadow quandle cocycle quivers $S Q_{X}^{S, \theta}(D, a)$ and $S Q_{X}^{S, \theta}\left(D^{\prime}, a\right)$ are isomorphic.

Proof. It is seen by the same argument with the proof that the quandle cocycle quiver is a link invariant given in $[6]$. 
In [6] a polynomial-valued link invariant, called the quiver enhanced cocycle polynomial, is introduced. The following is the shadow version.

Definition 5.6. Let $X$ be a finite quandle, $a$ an element of $X, S$ a subset of $\operatorname{End}(X), A$ an abelian group and $\theta: X^{3} \rightarrow A$ a 3-cocycle. The quiver enhanced shadow cocycle polynomial of an oriented link diagram $D$ is the polynomial

$$
\Phi_{X}^{S, \theta}(D, a)=\sum_{(v, w): \text { an edge of } S Q_{X}^{S, \theta}(D, a)} s^{\rho(v)} t^{\rho(w)}
$$

where the summation is taken over all edges $(v, w)$ of $S Q_{X}^{S, \theta}(D, a)$.

Obviously, this polynomial is a link invariant.

Example 5.7. The knots $4_{1}$ and $5_{1}$ are not distinguished by the quandle coloring quivers using the dihedral quandle $R_{5}$ of order 5 and any subset $S$ of $\operatorname{End}\left(R_{5}\right)$, since $\left|\mathrm{Col}_{R_{5}}\left(4_{1}\right)\right|=\left|\mathrm{Col}_{R_{5}}\left(5_{1}\right)\right|=25$ (Theorem 3.3).

Moreover, they are not distinguished by the quandle cocycle quivers using $R_{5}$, any subset $S$ of $\operatorname{End}\left(R_{5}\right)$ and any 2-cocycle $\theta: X^{2} \rightarrow A$, since any 2cocycle of $R_{5}$ is a coboundary (in fact $H^{2}\left(R_{5} ; A\right)=0,[2]$ ) and the weight given to each vertex of the quiver is $0 \in A$. Thus the quandle cocycle quiver is the quandle coloring quiver whose vertices are labeled with 0 .

On the other hand, these knots are distinguished by shadow quandle cocycle quivers. The shadow quandle cocycle quivers $S Q_{R_{p}}^{S, \theta_{p}}\left(4_{1}, 0\right)$ and $S Q_{R_{p}}^{S, \theta_{p}}\left(5_{1}, 0\right)$ using $R_{5}$, Mochizuki's 3-cocycle $\theta_{5}$ and $S=\{f\}$ where $f: R_{5} \rightarrow R_{5}$ is defined by $f(x)=x+2$, are as in Figures 3 and 4 .

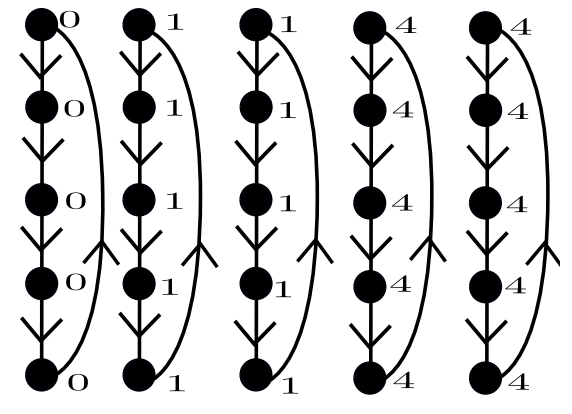

Figure 3: $S Q_{R_{p}}^{S, \theta_{p}}\left(4_{1}, 0\right)$

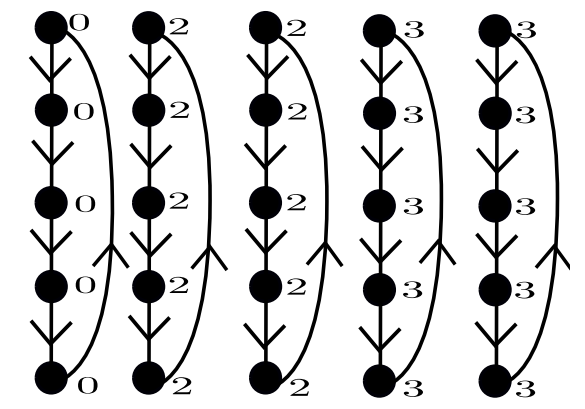

Figure 4: $S Q_{R_{p}}^{S, \theta_{p}}\left(5_{1}, 0\right)$ 
The quiver enhanced shadow cocycle polynomials are given as follows.

$$
\begin{aligned}
& \Phi_{R_{5}}^{S, \theta_{5}}\left(4_{1}, 0\right)=5+10 s t+10 s^{4} t^{4} \\
& \Phi_{R_{5}}^{S, \theta_{5}}\left(5_{1}, 0\right)=5+10 s^{2} t^{2}+10 s^{3} t^{3} .
\end{aligned}
$$

\section{The shadow quandle cocycle quivers using Mochizuki's 3-cocycle}

In this section, we discuss the shadow quandle cocycle quivers using Mochizuki's 3-cocycle. By Theorem 3.3, two different links which have the same $R_{p^{-}}$ coloring number can not be distinguished by the quandle coloring quivers using $R_{p}$.

In the case of the shadow quandle cocycle quiver, we have a similar result.

Theorem 6.1. Let $D$ and $D^{\prime}$ be oriented link diagrams, $p$ be an odd prime and $\theta_{p}$ be Mochizuki's 3-cocycle. For any $a \in R_{p}$ and $S \subset \operatorname{End}\left(R_{p}\right)$, the shadow quandle cocycle invariants $S Q_{R_{p}}^{S, \theta_{p}}(D, a)$ and $S Q_{R_{p}}^{S, \theta_{p}}\left(D^{\prime}, a\right)$ are isomorphic if and only if $\Phi_{\theta_{p}}(D)=\Phi_{\theta_{p}}\left(D^{\prime}\right)$.

Note that the set of shadow $R_{p}$-colorings of an oriented link diagram $D$ is a vector space over $\mathbb{Z}_{p}$. It is known that $\Phi_{\theta_{p}}(D)$ is characterized by the following lemmas.

Lemma $6.2([1])$. Let $c_{*}$ and $c_{*}^{\prime}$ be shadow $R_{p}$-colorings of an oriented link diagram $D$ such that $\left.c_{*}\right|_{\operatorname{Arc}(D)}=\left.c_{*}^{\prime}\right|_{\operatorname{Arc}(D)}$. Then it holds that $\Phi_{\theta_{p}}\left(D, c_{*}\right)=$ $\Phi_{\theta_{p}}\left(D, c_{*}^{\prime}\right)$.

Lemma $6.3([11])$. Let $c_{*}$ be a shadow $R_{p}$-coloring of an oriented link diagram $D$. Then it holds that $\Phi_{\theta_{p}}\left(D, a c_{*}\right)=a^{2} \Phi_{\theta_{p}}\left(D, c_{*}\right)$ for any $a \in \mathbb{Z}_{p}$.

Lemma $6.4([11])$. Let $c_{*}$ and $c_{*, 0}$ be shadow $R_{p}$-colorings of an oriented link diagram $D$ such that $\left.c_{*, 0}\right|_{\operatorname{Arc}(D)}$ is a trivial coloring. Then it holds that $\Phi_{\theta_{p}}\left(D, c_{*}\right)=\Phi_{\theta_{p}}\left(D, c_{*}+c_{*, 0}\right)$.

Proof of Theorem 6.1. The only if part is trivial. We show the if part. By Lemma 6.2, we have $\left\{\Phi_{\theta_{p}}\left(D, c_{*}\right) \mid c_{*} \in \operatorname{SCol}_{R_{p}}(D, a)\right\}=\left\{\Phi_{\theta_{p}}\left(D, c_{*}\right) \mid c_{*} \in\right.$ $\left.\mathrm{SCol}_{R_{p}}(D, b)\right\}$ for any $a, b \in R_{p}$. Therefore, $\Phi_{\theta_{p}}(D)=p\left\{\Phi_{\theta_{p}}\left(D, c_{*}\right) \mid c_{*} \in\right.$ $\left.\mathrm{SCol}_{R_{p}}(D, a)\right\}$ (cf. 11$)$. 
We construct a bijection $\Psi: \operatorname{SCol}_{R_{p}}(D, a) \rightarrow \operatorname{SCol}_{R_{p}}\left(D^{\prime}, a\right)$ as follow:

Let $\varphi: \operatorname{Col}_{R_{p}}(D) \rightarrow \mathrm{SCol}_{R_{p}}(D, a)$ and $\varphi^{\prime}: \operatorname{Col}_{R_{p}}\left(D^{\prime}\right) \rightarrow \mathrm{SCol}_{R_{p}}\left(D^{\prime}, a\right)$ be the natural bijections as in the proof of Proposition 5.2.

Firstly, put $V_{0}=\left\{c_{*} \in \operatorname{SCol}_{X}(D, a)\left|c_{*}\right|_{\operatorname{Arc}(D)}\right.$ is a trivial coloring $\}$ and $V_{0}^{\prime}=\left\{c_{*}^{\prime} \in \operatorname{SCol}_{X}\left(D^{\prime}, a\right)\left|c_{*}^{\prime}\right|_{\operatorname{Arc}\left(D^{\prime}\right)}\right.$ is a trivial coloring $\}$. We define a map $\Psi_{0}: V_{0} \rightarrow V_{0}^{\prime}$ by $\Psi_{0}\left(c_{*}\right)\left(x^{\prime}\right)=c_{*}(x)$ for any $x \in \operatorname{Arc}(D)$ and $x^{\prime} \in \operatorname{Arc}\left(D^{\prime}\right)$.

Next, take an element $c_{*, 1} \in \operatorname{SCol}_{R_{p}}(D, a) \backslash V_{0}$ and fix it. By assumption, there is a shadow $R_{p}$-coloring $c_{*, 1}^{\prime} \in \operatorname{SCol}_{R_{p}}\left(D^{\prime}, a\right) \backslash V_{0}^{\prime}$ such that $\Phi_{\theta_{p}}\left(D, c_{*, 1}\right)=$ $\Phi_{\theta_{p}}\left(D^{\prime}, c_{*, 1}^{\prime}\right)$. Let $c_{1}$ and $c_{1}^{\prime}$ be the shadow colorings of $D$ and $D^{\prime}$ respectively such that for any $x \in \operatorname{Arc}(D)$ and $x^{\prime} \in \operatorname{Arc}\left(D^{\prime}\right), c_{1}(x)=c_{1}^{\prime}\left(x^{\prime}\right)=1$ and $c_{1}\left(r_{\infty}\right)=c_{1}^{\prime}\left(r_{\infty}\right)=a$.

Put $V_{1}=\left\{\varphi\left(f \circ \varphi^{-1}\left(c_{*, 1}\right)\right) \mid f \in \operatorname{Aut}\left(R_{p}\right)\right\}$ and $V_{1}^{\prime}=\left\{\varphi^{\prime}\left(f \circ\left(\varphi^{\prime}\right)^{-1}\left(c_{*, 1}^{\prime}\right)\right) \mid\right.$ $\left.f \in \operatorname{Aut}\left(R_{p}\right)\right\}$. We define a map $\Psi_{1}: V_{0} \cup V_{1} \rightarrow V_{0}^{\prime} \cup V_{1}^{\prime}$ by $\left.\Psi_{1}\right|_{V_{0}}=\Psi_{0}$ and $\Psi_{1}\left(\varphi\left(f \circ \varphi^{-1}\left(c_{*, 1}\right)\right)\right)=\varphi^{\prime}\left(f \circ\left(\varphi^{\prime}\right)^{-1}\left(c_{*, 1}^{\prime}\right)\right)$ for any $f \in \operatorname{Aut}\left(R_{p}\right)$. Since $f$ is an automorphism and $c_{*, 1}^{\prime}$ is a nontirivial coloring, $\Psi_{1}$ is a bijection. Let $f$ be a quandle automorphism of $R_{p}$. By definition of $\varphi$, we have

$$
\begin{aligned}
\left.\varphi\left(f \circ \varphi^{-1}\left(c_{*, 1}\right)\right)\right|_{\operatorname{Arc}(D)} & =\left.f \circ c_{*, 1}\right|_{\operatorname{Arc}(D)} \\
& =\left.\left(a_{f} c_{*, 1}+b_{f} c_{1}\right)\right|_{\operatorname{Arc}(D)}
\end{aligned}
$$

where $a_{f} \in \mathbb{Z}_{p}^{\times}$and $b_{f} \in \mathbb{Z}_{p}$ are determined from $f$ by Lemma 3.4 (Remark 3.5) with $f(x)=a_{f} x+b_{f}$. By Lemmas 6.2, 6.3 and 6.4, it holds that

$$
\begin{aligned}
\Phi_{\theta_{p}}\left(D, \varphi\left(f \circ \varphi^{-1}\left(c_{*, 1}\right)\right)\right) & =\Phi_{\theta_{p}}\left(D, a_{f} c_{*, 1}+b_{f} c_{1}\right) \\
& =\left(a_{f}\right)^{2} \Phi_{\theta_{p}}\left(D, c_{*, 1}\right) .
\end{aligned}
$$

Similarly, we have $\Phi_{\theta_{p}}\left(D^{\prime}, \varphi^{\prime}\left(f \circ\left(\varphi^{\prime}\right)^{-1}\left(c_{*, 1}^{\prime}\right)\right)\right)=\left(a_{f}\right)^{2} \Phi_{\theta_{p}}\left(D^{\prime}, c_{*, 1}^{\prime}\right)$. By assumption, we see that

$$
\begin{aligned}
\Phi_{\theta_{p}}\left(D, \varphi\left(f \circ \varphi^{-1}\left(c_{*, 1}\right)\right)\right) & =\left(a_{f}\right)^{2} \Phi_{\theta_{p}}\left(D, c_{*, 1}\right) \\
& =\left(a_{f}\right)^{2} \Phi_{\theta_{p}}\left(D^{\prime}, c_{*, 1}^{\prime}\right) \\
& =\Phi_{\theta_{p}}\left(D^{\prime}, \varphi^{\prime}\left(f \circ\left(\varphi^{\prime}\right)^{-1}\left(c_{*, 1}^{\prime}\right)\right)\right) .
\end{aligned}
$$

Then it holds that $\left\{\Phi_{\theta_{p}}\left(D, c_{*}\right) \mid c_{*} \in V_{0} \cup V_{1}\right\}=\left\{\Phi_{\theta_{p}}\left(D^{\prime}, c_{*}^{\prime}\right) \mid c_{*}^{\prime} \in V_{0}^{\prime} \cup V_{1}^{\prime}\right\}$.

Suppose that $\mathrm{SCol}_{R_{p}}(D, a) \backslash\left(V_{0} \cup V_{1}\right) \neq \emptyset$. Take an element $c_{*, 2}$ of $\operatorname{SCol}_{R_{p}}(D, a) \backslash\left(V_{0} \cup\right.$ $\left.V_{1}\right)$ and fix it. There is a shadow $R_{p}$-coloring $c_{*, 2}^{\prime} \in \operatorname{SCol}_{R_{p}}(D, a) \backslash\left(V_{0}^{\prime} \cup V_{1}^{\prime}\right)$ such that $\Phi_{\theta_{p}}\left(D, c_{*, 2}\right)=\Phi_{\theta_{p}}\left(D^{\prime}, c_{*, 2}^{\prime}\right)$. Put $V_{2}=\left\{\varphi\left(f \circ \varphi^{-1}\left(c_{*, 2}\right)\right) \mid f \in\right.$ $\left.\operatorname{Aut}\left(R_{p}\right)\right\}$ and $V_{2}^{\prime}=\left\{\varphi^{\prime}\left(f \circ\left(\varphi^{\prime}\right)^{-1}\left(c_{*, 2}^{\prime}\right)\right) \mid f \in \operatorname{Aut}\left(R_{p}\right)\right\}$. Then, we define a bijection $\Psi_{2}: V_{0} \cup V_{1} \cup V_{2} \rightarrow V_{0}^{\prime} \cup V_{1}^{\prime} \cup V_{2}^{\prime}$ by $\left.\Psi_{1}\right|_{V_{0} \cup V_{1}}=\Psi_{1}$ 
and $\Psi_{2}\left(\varphi\left(f \circ \varphi^{-1}\left(c_{*, 2}\right)\right)\right)=\varphi^{\prime}\left(f \circ\left(\varphi^{\prime}\right)^{-1}\left(c_{*, 2}^{\prime}\right)\right)$ for any automorphism $f \in$ $\operatorname{Aut}\left(R_{p}\right)$. Repeating this proceedure until the domain of $\Psi_{n}$ is $\operatorname{SCol}_{R_{p}}(D, a)$, we have a bijection $\Psi=\Psi_{n}: \operatorname{SCol}_{R_{p}}(D, a) \rightarrow \operatorname{SCol}_{R_{p}}\left(D^{\prime}, a\right)$ such that $\Phi_{\theta_{p}}\left(D, c_{*}\right)=\Phi_{\theta_{p}}\left(D^{\prime}, \Psi\left(c_{*}\right)\right)$ for any $c_{*} \in \operatorname{SCol}_{R_{p}}(D, a)$. By Remark 3.5. $\Psi$ is a quiver isomorphism between $S Q_{R_{p}}^{S}(D, a)$ and $S Q_{R_{p}}^{S}\left(D^{\prime}, a\right)$ for any subset $S \subset \operatorname{End}\left(R_{p}\right)$.

\section{Acknowledgements}

The author would like to thank Seiichi Kamada and Hirotaka Akiyoshi for helpful advice and discussions on this research.

\section{References}

[1] J S. Carter, D. Jelsovsky, S. Kamada, L. Langford, and M. Saito, Quandle cohomology and state-sum invariants of knotted curves and surfaces, Trans. Amer. Math. Soc. 355 (2003), no. 10, 3947-3989.

[2] J S. Carter, D. Jelsovsky, S. Kamada, and M. Saito, Computations of quandle cocycle invariants of knotted curves and surfaces, Adv. Math. 157 (2001), no. 1, 36-94.

[3] J S. Carter, S. Kamada, and M. Saito, Geometric interpretations of quandle homology, J. Knot Theory Ramifications 10 (2001), no. 03, 345-386.

[4] _ Surfaces in 4-space, Encyclopaedia of Mathematical Sciences, Vol 142, LowDimensional Topology, III, Springer-Verlag, Berlin Heidelberg New York, 2004.

[5] K. Cho and S. Nelson, Quandle coloring quivers, J. Knot Theory Ramifications 28 (2019), no. 01, 1950001 (12 pages).

[6] _ Quandle cocycle quivers, Topology Appl. 268 (2019), 106908 (10 pages).

[7] D. Joyce, A classifying invariant of knots, the knot quandle, J. Pure Appl. Algebra 23 (1982), no. 1, 37-65.

[8] S. V. Matveev, Distributive groupoids in knot theory, Mat. Sb. 161 (1982), no. 1, $78-88$.

[9] T. Mochizuki, Some calculations of cohomology groups of finite Alexander quandles, J. Pure Appl. Algebra 179 (2003), no. 3, 287-330.

[10] - The third cohomology groups of dihedral quandles, J. Knot Theory Ramifications 20 (2011), no. 07, 1041-1057.

[11] S. Satoh, A note on the shadow cocycle invariant of a knot with a base point, J. Knot Theory Ramifications 16 (2007), no. 07, 959-967. 